\title{
Was the Universe Reionized at Redshift 10?
}

\section{Citation}

Loeb, Abraham, Rennan Barkana, and Lars Hernquist. 2005. "Was the Universe Reionized at Redshift 10?" The Astrophysical Journal 620 (2): 553-58. https://doi.org/10.1086/427229.

\section{Permanent link}

http://nrs.harvard.edu/urn-3:HUL.InstRepos:41381645

\section{Terms of Use}

This article was downloaded from Harvard University's DASH repository, and is made available under the terms and conditions applicable to Other Posted Material, as set forth at http:// nrs.harvard.edu/urn-3:HUL.InstRepos:dash.current.terms-of-use\#LAA

\section{Share Your Story}

The Harvard community has made this article openly available.

Please share how this access benefits you. Submit a story.

Accessibility 


\title{
Was the Universe Reionized at Redshift 10?
}

\author{
Abraham Loeb \\ Astronomy Department, Harvard University, 60 Garden Street, Cambridge, MA 02138; \\ aloeb@cfa.harvard.edu \\ Rennan Barkana \\ School of Physics and Astronomy, The Raymond and Beverly Sackler Faculty of Exact Sciences, \\ Tel Aviv University, Tel Aviv 69978, ISRAEL; barkana@wise.tau.ac.il \\ Lars Hernquist \\ Astronomy Department, Harvard University, 60 Garden Street, Cambridge, MA 02138; \\ lars@cfa.harvard.edu
}

\begin{abstract}
Recently, Pello et al. (2004) claimed to have discovered a galaxy at a redshift $z=10$, and identified a feature in its spectrum with a hydrogen Ly $\alpha$ emission line. If this identification is correct, we show that the intergalactic medium (IGM) around the galaxy must be significantly ionized; otherwise, the damping wing of Ly $\alpha$ absorption by the neutral IGM would have greatly suppressed the emission line. We find either that the large-scale region surrounding this galaxy must have been largely reionized by $z=10$ (with a neutral fraction $\lesssim 0.4$ ) or that the stars within the galaxy must be massive ( $\gtrsim 100 M_{\odot}$ ), and hence capable of producing a sufficiently large H II region around it. We generalize these conclusions and derive the maximum Ly $\alpha$ line flux for a given UV continuum flux of galaxies prior to the epoch of reionization.
\end{abstract}

Key Words: galaxies: high-redshift, cosmology: theory, galaxies: formation

\section{Introduction}

Pello et al. (2004) have reported the discovery of a galaxy at a redshift $z=10$, which is gravitationally magnified by a factor $\sim 25-100$ by the foreground galaxy cluster Abel 1835 . The redshift identification is supported by the spectroscopic detection of an emission line at $1.338 \mu \mathrm{m}$, the photometric signature of an absorption trough at shorter wavelengths, and the lensing geometry. Here, we examine the far-reaching implications of associating this spectral feature with the Ly $\alpha$ emission line from a galaxy at $z=10$. In particular, we show that if hydrogen in the intergalactic medium (IGM) around this galaxy were neutral, then the damping wing of its Ly $\alpha$ absorption would have greatly suppressed the $\operatorname{Ly} \alpha$ emission line of the galaxy. Our results provide a strong 
incentive for obtaining higher quality data on this galaxy. More generally, we derive the maximum Ly $\alpha$ line flux for a given UV continuum flux of galaxies prior to the epoch of reionization. Future detection of bright Ly $\alpha$ lines from high-redshift galaxies can be used to improve the current lower limit on the redshift of reionization.

Throughout, we adopt values for cosmological parameters derived from recent observations of the cosmic microwave background (Spergel et al. 2003; Seljak et al. 2003). For the contributions to the energy density, we assume ratios relative to the critical density of $\Omega_{m}=0.27, \Omega_{\Lambda}=0.73$, and $\Omega_{b}=0.046$, for matter, vacuum energy, and baryons, respectively. We also choose a Hubble constant $H_{0}=72 \mathrm{~km} \mathrm{~s}^{-1} \mathrm{Mpc}^{-1}$, and a primordial scale-invariant $(n=1)$ power-law power spectrum with $\sigma_{8}=0.9$, where $\sigma_{8}$ is the root-mean-square amplitude of mass fluctuations in spheres of radius $8 h^{-1} \mathrm{Mpc}$.

\section{Basic Absorption Parameters}

The absorption profile owing to H I in the IGM depends on the redshift $z_{S}$ and halo mass $M$ of the source, and parameters that characterize the production of ionizing photons by the galaxy. The first parameter, $d N_{\gamma} / d t$, is the total rate at which hydrogen ionizing photons from the galaxy enter the IGM. The second is the age of the source, $t_{S}$, namely the period of time during which the source has been active (assuming a steady $d N_{\gamma} / d t$ during that time, for simplicity).

We consider ages in the range $t_{S} \sim 10^{7}-10^{8} \mathrm{yr}$, comparable to the dynamical time within the host galaxies of interest. For given values of $M$ and $t_{S}$, we find it useful to express $d N_{\gamma} / d t$ as follows:

$$
\frac{d N_{\gamma}}{d t}=\frac{M}{m_{p}} \frac{\Omega_{b}}{\Omega_{m}} \frac{N_{\text {ion }}}{t_{S}}
$$

where $m_{p}$ is the proton mass, and $N_{\text {ion }}$ gives the overall number of ionizing photons per baryon in the galaxy (ignoring helium). To derive the expected range of possible values for $N_{\text {ion }}$, we note that it is determined by

$$
N_{\text {ion }}=N_{\gamma} f_{*} f_{\text {esc }},
$$

where we assume that baryons are incorporated into stars with an efficiency of $f_{*}$, that $N_{\gamma}$ ionizing photons are produced per baryon in stars, and that the escape fraction (into the IGM) for the resulting ionizing radiation is $f_{\text {esc }}$. When necessary, we adopt $f_{*}=10 \%$ (consistent with a comparison of numerical simulations to the observed cosmic star formation rate at low redshift [Hernquist \& Springel 2003]), and consider $f_{\text {esc }}$ in the full range 0-1. As explained below, our main conclusions are insensitive to our choices for the values of $f_{*}$ and $f_{\text {esc }}$.

The parameter $N_{\gamma}$ depends on the initial mass function (IMF) of the stars. In what follows, we consider two examples. The first is the locally-measured IMF of Scalo (1998), which yields [using Leitherer et al. (1999)] $N_{\gamma}=4300$ for a metallicity of $1 / 20$ of the solar value. The second is an extreme Pop III IMF, assumed to consist entirely of zero metallicity $M \gtrsim 100 M_{\odot}$ stars, 
which yields $N_{\gamma}=44000$ based on the ionization rate per star, the stellar spectrum, and the mainsequence lifetime of these stars (Bromm, Kudritzki, \& Loeb 2001). Note that the star formation rate (hereafter SFR) corresponding to eq. (1) is

$$
\mathrm{SFR}=0.171\left(\frac{M}{10^{9} M_{\odot}}\right)\left(\frac{t_{S}}{10^{8} \mathrm{yr}}\right)^{-1}\left(\frac{f_{*}}{0.1}\right)\left(\frac{\Omega_{b} / \Omega_{m}}{0.17}\right) M_{\odot} \mathrm{yr}^{-1} .
$$

\section{Ionization and the Damping Wing}

An ionizing source embedded within the neutral IGM can ionize a region of maximum physical size

$$
R_{\max }=0.138\left(\frac{M}{10^{9} M_{\odot}}\right)^{1 / 3}\left(\frac{1+z}{11}\right)^{-1}\left(\frac{\Omega_{m} h^{2}}{0.14}\right)^{-1 / 3}\left(\frac{N_{\gamma} f_{*}}{430}\right)^{1 / 3} f_{\mathrm{esc}}^{1 / 3} \mathrm{Mpc},
$$

assuming that recombinations are negligible and that all ionizing photons are absorbed by hydrogen atoms. In this Letter we conservatively adopt this maximum size, since a smaller region would only strengthen our main conclusions (for more elaborate discussions, see Haiman 2002; Santos 2003).

When dealing with $\operatorname{Ly} \alpha$ emission and absorption, it is useful to associate a redshift $z$ with a photon of observed wavelength $\lambda_{\text {obs }}$ according to $\lambda_{\text {obs }}=\lambda_{\alpha}(1+z)$, where the Ly $\alpha$ wavelength is

$\lambda_{\alpha}=1215.67 \AA$. The optical depth $\tau_{\text {damp }}$ owing to the IGM damping wing of neutral gas between $z_{1}$ and $z_{2}$ (where $z_{1}<z_{2}<z$ ) can be calculated analytically (Miralda-Escudé 1998). For a source above the reionization redshift, $z_{2}$ is the redshift corresponding to the blue edge of its $\mathrm{H}$ II region, and $z_{1}$ is the reionization redshift. We define $\Delta \lambda=\lambda_{\alpha}\left(z-z_{2}\right)$, which is related to the physical distance $\Delta D$ between the source and the H II region edge by

$$
\Delta D=0.164\left(\frac{\Delta \lambda}{10 \AA}\right)\left(\frac{1+z}{11}\right)^{-5 / 2}\left(\frac{\Omega_{m} h^{2}}{0.14}\right)^{-1 / 2} \mathrm{Mpc} .
$$

In the limit where $z_{2}$ is very close to $z$ while $z_{1}$ is much farther away, the damping optical depth can be accurately approximated as

$$
\tau_{\text {damp }} \approx \frac{1.16 \mathrm{Mpc}}{\Delta D}\left(\frac{\Omega_{b} / \Omega_{m}}{0.17}\right)\left\{1-\frac{\Delta \lambda}{2.96 \times 10^{3} \AA} \frac{11}{1+z} \log \left[\frac{2.68 \times 10^{4} \AA}{\Delta \lambda} \frac{1+z}{11}\right]\right\},
$$

which is independent of $z_{1}$.

\section{Ly $\alpha$-emitting Galaxies}

Next we discuss the use of Ly $\alpha$-emitting galaxies to probe reionization. To study the detectability of high-redshift galaxies, we must convert the SFR of a galaxy in a halo of a given mass [see eq. (3)] to a flux level for both the Ly $\alpha$ line and the UV continuum. Assuming that 2/3 of the ionizing photons that are reprocessed (i.e., that do not escape from the galaxy) lead to a 
hydrogen atom in the $2 p$ state and thus result in the emission of a Ly $\alpha$ photon (Osterbrock 1974), the rest-frame conversion factor between the SFR and the luminosity of the Ly $\alpha$ line is

$$
L(\operatorname{Ly} \alpha)=1.8 \times 10^{42}\left(1-f_{\mathrm{esc}}\right)\left(\frac{N_{\gamma}}{4300}\right)\left(\frac{\mathrm{SFR}}{1 M_{\odot} \mathrm{yr}^{-1}}\right) \mathrm{erg} \mathrm{s}^{-1},
$$

where this conversion is valid for any $t_{S} \geq 10^{7} \mathrm{yr}$ (since the high-mass, short-lived stars dominate the ionizing intensity). Using the photon production rate in Leitherer et al. (1999) for the Scalo (1998) IMF, and the stellar spectrum and lifetime in Bromm et al. (2001) for the extreme Pop III IMF, we find that the continuum flux can be converted (in the rest frame) as follows, depending on $t_{S}$ and source rest-frame wavelength $\lambda$ :

$$
F_{\nu}(\lambda)=10^{27} N_{\text {cont }}\left(\frac{\mathrm{SFR}}{1 M_{\odot} \mathrm{yr}^{-1}}\right) \mathrm{erg} \mathrm{s}^{-1} \mathrm{~Hz}^{-1}
$$

where

$$
N_{\text {cont }}= \begin{cases}10,, & \text { for a Scalo (1998) IMF, } t_{S}=10^{8} \mathrm{yr}, \lambda=1500 \AA \\ 8.7, & \text { for a Scalo (1998) IMF, } t_{S}=10^{8} \mathrm{yr}, \lambda=2000 \AA \\ 5.5, & \text { for a Scalo (1998) IMF, } t_{S}=10^{7} \mathrm{yr}, \lambda=1500 \AA \\ 4.6, & \text { for a Scalo (1998) IMF, } t_{S}=10^{7} \mathrm{yr}, \lambda=2000 \AA \\ 5.9, & \text { for an extreme Pop III IMF, } t_{S} \geq 3 \times 10^{6} \mathrm{yr}, \lambda=1500 \AA \\ 3.8, & \text { for an extreme Pop III IMF, } t_{S} \geq 3 \times 10^{6} \mathrm{yr}, \lambda=2000 \AA .\end{cases}
$$

Note that for a given SFR and $f_{\text {esc }}$, an extreme Pop III population produces a Ly $\alpha$ line $\sim 10$ times more luminous than does a Scalo (1998) IMF, but the UV continuum is comparable for the two IMFs. The reason is that high-mass, short-lived stars strongly dominate the production of ionizing photons, but in the Scalo IMF case, lower-mass and longer-lived stars contribute substantially to the continuum.

To evaluate the impact of absorption on the $\operatorname{Ly} \alpha$ line, we require an estimate of the width of the line. Assuming a Gaussian profile, we estimate the one-dimensional velocity dispersion $\sigma_{v}$ by $\sigma_{v} \approx V_{c} / \sqrt{2}$, where $V_{c}$ is the virial circular velocity of the halo; this yields

$$
\sigma_{v}=26.9\left(\frac{M}{10^{9} M_{\odot}}\right)^{1 / 3}\left(\frac{1+z}{11}\right)^{1 / 2}\left(\frac{\Omega_{m} h^{2}}{0.14}\right)^{1 / 6} \mathrm{~km} \mathrm{~s}^{-1} .
$$

The observed scale-length $\sigma_{\lambda}$ is related to $\sigma_{v}$ by

$$
\sigma_{v}=22.4\left(\frac{\sigma_{\lambda}}{1 \AA}\right)\left(\frac{1+z}{11}\right)^{-1} \mathrm{~km} \mathrm{~s}^{-1} .
$$

\section{Results}

Identifying the redshifted Ly $\alpha$ line based on its properties and a strong photometric continuum break, Pello et al. (2004) measured an amplified line flux for the candidate $z=10.0$ galaxy of 
$(4.1 \pm 0.5) \times 10^{-18} \mathrm{erg} \mathrm{cm}^{-2} \mathrm{~s}^{-1}$ and a UV continuum flux density of $\left(3.6_{-0.7}^{+1.0}\right) \times 10^{-30} \mathrm{erg} \mathrm{cm}^{-2}$ $\mathrm{s}^{-1} \mathrm{~Hz}^{-1}$ averaged over the $1360-1640 \AA$ rest-frame band, and $\left(2.3_{-0.7}^{+0.9}\right) \times 10^{-30} \mathrm{erg} \mathrm{cm}^{-2} \mathrm{~s}^{-1} \mathrm{~Hz}^{-1}$

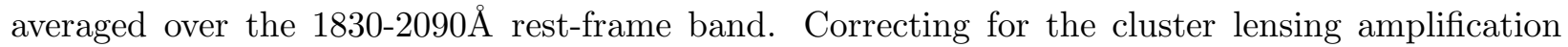
factor of $\sim 25$, they estimated a SFR of 2-3 $M_{\odot} \mathrm{yr}^{-1}$ based on the continuum strength and 0.03$0.09 M_{\odot} \mathrm{yr}^{-1}$ based on the line flux. This discrepancy, they suggested, indicates a factor of $\sim 40$ absorption of the line, in principle owing to both $\mathrm{H}$ I absorption and dust extinction, but in this case they noted that the steep UV slope indicates negligible extinction. The observed line is somewhat resolved, allowing Pello et al. (2004) to place an upper limit of $\sim 200 \mathrm{~km} / \mathrm{s}$ on $\sigma_{v}$, with best fits obtained for $\sigma_{v}<60 \mathrm{~km} / \mathrm{s}$.

We reconsider the interpretation of the observations of Pello et al. (2004), accounting for different possible IMFs and source ages. We find a luminosity distance to the galaxy of $105 \mathrm{Gpc}$, yielding a rest-frame luminosity (demagnified by a factor of 25) of $2.2 \times 10^{41} \mathrm{erg} \mathrm{s}^{-1}$ in the line and a (demagnified) rest-frame luminosity density of $1.7 \times 10^{28} \mathrm{erg} \mathrm{s}^{-1} \mathrm{~Hz}^{-1}$ and $1.1 \times 10^{28} \mathrm{erg}$ $\mathrm{s}^{-1} \mathrm{~Hz}^{-1}$ in the two UV continuum ranges, respectively (including the extra redshift factor for frequency bands). Estimating the overall conversion factor with the values for $\lambda=1500 \AA$ and for $\lambda=2000 \AA$, respectively, we find using eq. (8) the SFR implied by the continuum flux, which then implies through eq. (7) an intrinsic Ly $\alpha$ line luminosity (before absorption). We also estimate a halo mass using eq. (3), assuming $f_{*}=0.1$.

Table 1 shows our derived parameters for the $z=10.0$ galaxy and its host halo, depending on the IMF and the source lifetime. The Table gives the intrinsic line luminosity expected in the absence of $\mathrm{H}$ I absorption or dust extinction; also given are the expected intrinsic $\sigma_{v}$ based on eq. (10), and the maximum physical size of the H II region based on eq. (4). Given the observations, the derived quantities given in the Table are independent of the star formation efficiency $f_{*}$, except for the estimated halo mass and $\sigma_{v}$. Note that the observed ratio of the values of the continuum flux in the two measured wavelength regions is $1.6 \pm 0.7$, compared to a predicted ratio of 1.2 for a Scalo IMF and 1.6 for a Pop III IMF. Thus, the steep UV slope is more consistent with a Pop III IMF, but the discrepancy with a Scalo IMF is not highly significant. In either case, this measurement suggests that dust extinction is not strong (compare discussion in Pello et al. 2004). For each case in the Table we average the SFR derived from the two UV continuum measurements. The errors in these two measurements introduce errors of $\sim 20 \%$ in the predicted Ly $\alpha$ line luminosity and $\sim 7 \%$ in the predicted $R_{\max }$.

For a given value of $f_{\text {esc }}$, the rest-frame Ly $\alpha$ line luminosity can be predicted from the UV continuum by accounting for the factor of $1-f_{\text {esc }}$ (which arises since ionizing photons produce Ly $\alpha$ only if they are absorbed within the galaxy and reprocessed), and adding the damping wing absorption which depends on $R_{\max }$ (which depends on $f_{\text {esc }}$ as well). For each case in the Table, we vary $f_{\text {esc }}$ over all possible values and find the maximum possible Ly $\alpha$ line luminosity $L_{\max }^{\alpha}$. If we allow a 2- $\sigma$ measurement error in the UV continuum flux, then the value of $L_{\max }^{\alpha}$ becomes, respectively for the four cases shown in Table 1, $2.1 \times 10^{39} \mathrm{erg} \mathrm{s}^{-1}, 2.6 \times 10^{40} \mathrm{erg} \mathrm{s}^{-1}, 1.4 \times 10^{42}$ $\mathrm{erg} \mathrm{s}^{-1}$, and $9.3 \times 10^{42} \mathrm{erg} \mathrm{s}^{-1}$. These numbers can be compared to the observed value of $2.2 \times 10^{41}$ 
$\operatorname{erg~s}^{-1}$ (with a $2-\sigma$ lower limit of $1.7 \times 10^{41} \mathrm{erg} \mathrm{s}^{-1}$ ). Thus, the Scalo (1998) IMF predicts a much weaker line than that observed and is ruled out, assuming that the IGM surrounding the H II region is neutral. An extreme Pop III IMF, however, is easily consistent with the observations even for a short burst $\left(t_{S}=10^{7} \mathrm{yr}\right)$.

Each line in the Table provides the mass of the source halo. Interestingly, numerical simulations indicate that the multiplicity function of star formation at $z \sim 10$ peaks at halo masses $\sim 10^{9} M_{\odot}$ (Springel \& Hernquist 2003), comparable to our inferred values. Using a halo mass function based on numerical simulations (Sheth \& Tormen 1999, 2002), we find that the mean physical separation between halos at or above the inferred mass is $0.21 \mathrm{Mpc}, 0.54 \mathrm{Mpc}, 0.21 \mathrm{Mpc}$, and $0.82 \mathrm{Mpc}$, respectively, for the four cases in the Table.

We have found that the Scalo (1998) IMF is inconsistent with the observations if the H II region is produced by the source galaxy and is surrounded by fully neutral IGM. There are two possible ways to resolve this inconsistency. One is to assume a larger surrounding H II region that is produced by the collective ionizing flux from nearby galaxies. The required minimum size for producing the observed line flux (with $f_{\text {esc }}=0$ in order to maximize the intrinsic line flux) is 0.35 $\operatorname{Mpc}\left(t_{S}=10^{7} \mathrm{yr}\right)$ or $0.43 \mathrm{Mpc}\left(t_{S}=10^{8} \mathrm{yr}\right)$. Given the above halo abundances, such a sphere will typically contain a number of equally or more massive halos, i.e., an average number of 20 and 2, respectively. Barkana \& Loeb (2004b) showed that the fluctuations in the number of halos in such a volume are much larger than Poisson fluctuations, owing to biased galaxy formation which greatly amplifies large-scale density perturbations. In particular, the typical 1- $\sigma$ fluctuation in the number of halos is a factor of 3 and 5 in these two cases, respectively; thus it is possible that the selection effect of the observability of the Ly $\alpha$ line has revealed a galaxy in a region that had been previously reionized, e.g., by a positive $2-\sigma$ fluctuation in the local number density of galaxies, while most of the rest of the universe remained neutral at that redshift.

Another possibility is that the H II region has the size given in the Table, but the IGM beyond the $\mathrm{H}$ II region is on average only partially ionized and thus produces a weaker damping wing than we have assumed thus far. If as before we maximize the flux over all possible values of $f_{\text {esc }}$, then

Table 1. Derived parameters for the Pello et al. (2004) galaxy (see text)

\begin{tabular}{lccccccc}
\hline \hline IMF & $\begin{array}{c}t_{S} \\
{[\mathrm{yr}]}\end{array}$ & $\begin{array}{c}\text { SFR } \\
{\left[M_{\odot} \mathrm{yr}^{-1}\right]}\end{array}$ & $\begin{array}{c}\text { Halo mass } \\
{\left[M_{\odot}\right]}\end{array}$ & $\begin{array}{c}\text { Ly } \alpha \text { luminosity } \\
{\left[\left(1-f_{\mathrm{esc}}\right) \mathrm{erg} \mathrm{s}^{-1}\right]}\end{array}$ & $\begin{array}{c}\sigma_{v} \\
{[\mathrm{~km} / \mathrm{s}]}\end{array}$ & $\begin{array}{c}R_{\max } \\
{\left[f_{\mathrm{esc}}^{1 / 3} \mathrm{Mpc}\right]}\end{array}$ & $\begin{array}{c}L_{\max }^{\alpha} \\
{\left[\mathrm{erg} \mathrm{s}^{-1}\right]}\end{array}$ \\
\hline Scalo & $10^{7}$ & 2.8 & $1.6 \times 10^{9}$ & $5.0 \times 10^{42}$ & 32 & 0.16 & $5.1 \times 10^{38}$ \\
Scalo & $10^{8}$ & 1.5 & $8.8 \times 10^{9}$ & $2.7 \times 10^{42}$ & 56 & 0.28 & $9.4 \times 10^{39}$ \\
Pop III & $10^{7}$ & 2.9 & $1.7 \times 10^{9}$ & $5.3 \times 10^{43}$ & 32 & 0.36 & $5.5 \times 10^{41}$ \\
Pop III & $10^{8}$ & 2.9 & $1.7 \times 10^{10}$ & $5.3 \times 10^{43}$ & 69 & 0.77 & $4.8 \times 10^{42}$ \\
\hline
\end{tabular}




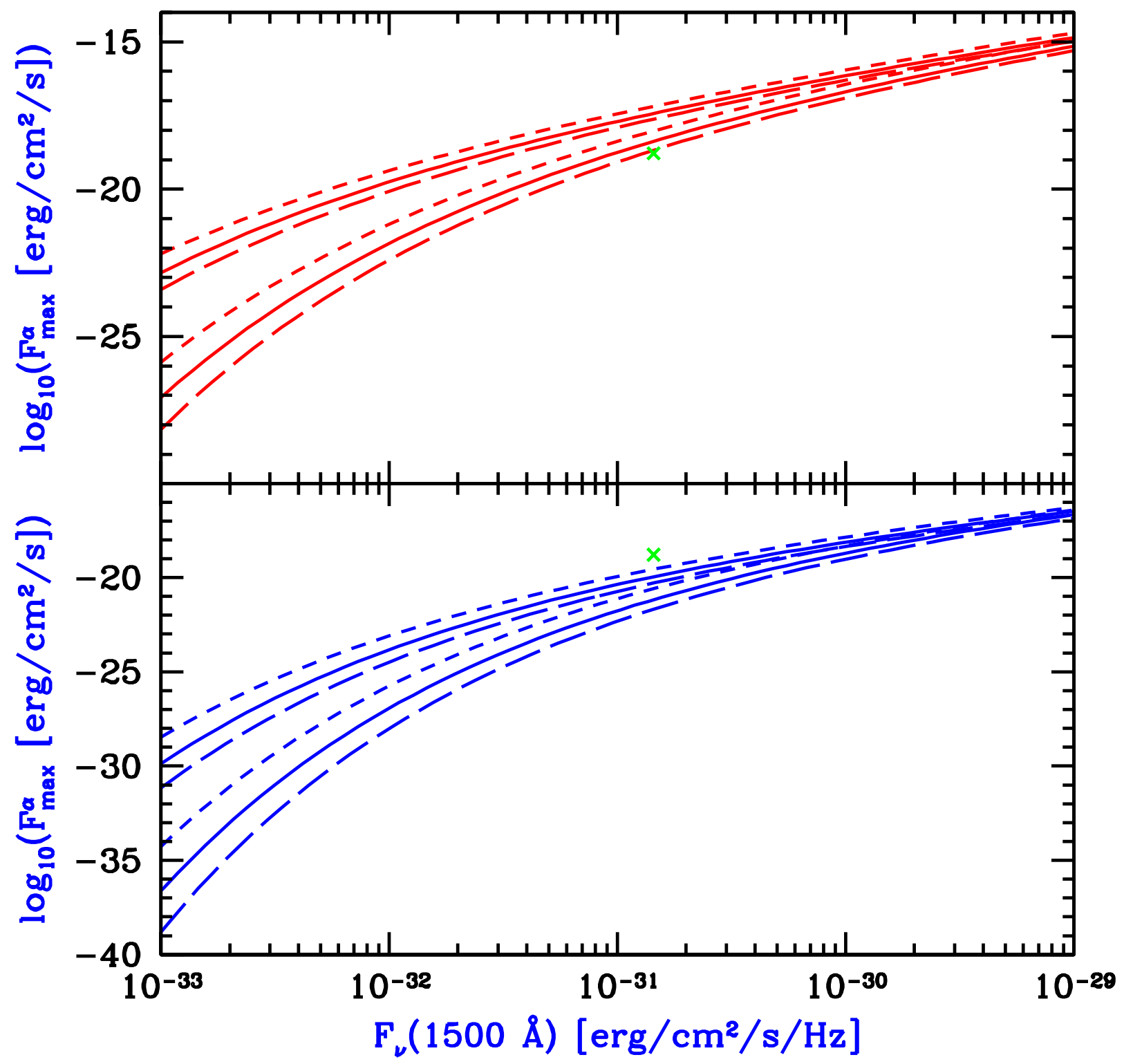

Fig. 1.- Maximum observable flux of the Ly $\alpha$ line versus UV continuum flux (at a rest-frame wavelength of $1500 \AA$ ) of a galaxy prior to reionization (i.e., assuming that the H II region of the galaxy is surrounded by a neutral IGM). We assume a narrow emission line $\left(\lesssim 100 \mathrm{~km} \mathrm{~s}^{-1}\right)$. We consider a Scalo (1998) IMF (bottom panel) or a Pop III IMF (top panel), at redshifts 7 (shortdashed curves), 10 (solid curves), or 13 (long-dashed curves). In each case, a pair of curves are shown, corresponding to $t_{S}=10^{8} \mathrm{yr}$ (higher) or $t_{S}=10^{7} \mathrm{yr}$ (lower). Also shown are the observed flux values for the Pello et al. (2004) $z=10$ galaxy, demagnified by a factor of 25 (denoted by $\times$ ). 
the maximum allowed neutral fraction is $0.27\left(t_{S}=10^{7} \mathrm{yr}\right)$ or $0.37\left(t_{S}=10^{8} \mathrm{yr}\right)$.

The damping wing of a neutral IGM limits the maximal flux of the Ly $\alpha$ line from any galaxy prior to reionization. Figure 1 illustrates this upper limit as a function of the UV continuum flux of that galaxy, which affects the size of the H II region around it. We have maximized the line flux over $f_{\text {esc }}$ and calculated the damping wing at the center of the line assuming that the IGM is neutral and that its opacity does not change significantly across the width of the line. The latter assumption is well satisfied for lines with velocity widths $\lesssim 100 \mathrm{~km} \mathrm{~s}^{-1}$. Our flux limits are independent of the star formation efficiency $f_{*}$. The galaxy discovered by Pello et al. (2004) (denoted by $\times$ ) should be compared to the solid lines (indicating results for $z=10$ ); its detected Ly $\alpha$ line flux requires that the IGM be ionized for a Scalo (1998) IMF (bottom panel) but is consistent with a neutral IGM for a Pop III IMF (top panel).

\section{Discussion}

Figure 1 shows that if the galaxy discovered by Pello et al. (2004) indeed has a redshift $z=10$, then either its stars are very massive $\left(\gtrsim 100 M_{\odot}\right)$ and hence capable of creating a large H II region around it, or the large-scale IGM around it has already been mostly reionized (with a neutral fraction $\lesssim 0.4)$. The ratio of the galaxy's UV continuum flux in two bands is consistent with the first possibility, but better determination of the continuum spectrum or detection of other recombination lines (Bromm, Kudritzki, \& Loeb 2001; Tumlinson, Giroux, \& Shull 2001; Oh, Haiman, \& Rees 2001) is required to make a convincing case. For example, a stellar population with a top-heavy IMF will produce substantial flux in He II Ly $\alpha(303.9 \AA)$ and He II $\lambda 1640$. A search for features in the spectrum of a redshift $z=10$ galaxy at $\lambda_{\text {obs }}=3343 \AA$ and $1.8 \mu \mathrm{m}$ would constrain the first interpretation. The discovery of other galaxies may test the second possibility, since the damping wing of a neutral IGM limits the maximum Ly $\alpha$ line flux of any galaxy prior to reionization based on its UV continuum flux.

The imprint of the damping wing is insensitive to a variety of complicating factors. Clumping of gas and its infall onto the galaxy have a minor effect, since the damping wing averages the IGM over large physical scales $(\sim 1 \mathrm{Mpc})$. Moreover, the characteristic size of the $\mathrm{H}$ II region around a bright galaxy at $z \sim 10$ is an order of magnitude larger than the scale of its infall region. The characteristic peculiar velocity of galaxies at $z \sim 10$ is an order of magnitude smaller than the Hubble velocity at the edge of their H II regions, and can only significantly change the effect of resonant absorption on the line profile. We have ignored resonant absorption from $\mathrm{H}$ I within the $\mathrm{H}$ II region since it would not suppress even the blue wing of the Ly $\alpha$ emission line if the galaxy had a peculiar velocity (in the direction of recession) larger than the line width (thus allowing the line to appear nearly symmetric); such a peculiar velocity may not be improbable within small samples because it makes the line more easily observable and adds a selection bias. In any case, the addition of resonant absorption would only strengthen our limits on the Ly $\alpha$ line flux before reionization. Dust extinction in the host galaxy would affect ionizing photons (and thus the Ly $\alpha$ line flux) more 
than it would the continuum at longer wavelengths. Based on the observed UV continuum, we would have predicted a weaker line with the inclusion of extinction, and so our argument would have been stronger.

We have considered the lowest (most probable) value within the range of lensing magnification factors inferred by Pello et al. (2004). A higher value would again strengthen our limits. For example, a magnification factor of 100 would imply that the line was 4 times weaker intrinsically, but the predicted line from the UV continuum would then be weakened by more than a factor of 4 since the smaller H II region would imply a damping wing that suppressed more of the line flux.

Current observational constraints on reionization provide an inconsistent picture. On the one hand, the large-scale polarization anisotropies of the cosmic microwave background measured by WMAP imply a reionization redshift of 10-20 (Kogut et al. 2003), while on the other hand, the extent of the $\mathrm{H}$ II regions around the highest redshift quasars indicates ${ }^{1}$ a significantly neutral IGM at $z \sim 6.4$ (Wyithe \& Loeb 2004). Moreover, the IGM should have been cooler than observed at $z \sim 3$-4 if hydrogen had been fully reionized at $z \gtrsim 9$ (Theuns et al. 2002; Hui \& Haiman 2003). Theoretically, it is possible that the ionization fraction evolved in a complex, non-monotonic fashion owing to an early episode of Pop III star formation (Wyithe \& Loeb 2003; Cen 2003).

Existing observations do not enable us to discriminate between the two interpretations of a positive detection of a Ly $\alpha$ emission line from the $z=10$ galaxy of Pello et al. (2004). Sokasian et al. $(2003 \mathrm{a}, \mathrm{b})$ have studied the process of reionization using cosmological simulations with radiative transfer; they have shown that for a Scalo-type IMF, reionization occurs over an extended redshift interval. For models in which the Ly $\alpha$ optical depth at $z \approx 6$ matches the value inferred from the SDSS quasars, a substantial fraction of the mass in the universe $(>30 \%)$ is already completely ionized by $z=10$. Since reionization proceeds "inside-out" affecting overdense regions first within the simulated volume (see also Gnedin 2000), we would expect the environment of the Pello et al. (2004) galaxy to be ionized. If the evolution had been more complex and included sources with population III stars prior to $z=12$, an even larger fraction of the mass would have been ionized by $z=10$ (Wyithe \& Loeb 2003; Sokasian et al. 2003b). This picture could change, however, if reionization occurred "outside-in" (e.g., Miralda-Escudé, Haehnelt \& Rees 2000). As shown by Barkana \& Loeb (2004), the fluctuations in the real universe should be far larger than indicated in existing simulations because of their limited box size. In reality, small fluctuations on scales larger than the box are greatly amplified through biased galaxy formation at high redshifts.

The various scenarios for reionization can be tested with more data on Ly $\alpha$ emitting galaxies (supplementing recent work by $\mathrm{Hu}$ et al. 2002, 2004; Malhotra \& Rhoads 2002; Kodaira et al. 2003; Santos et al. 2003; Kneib et al. 2004; Stanway et al. 2004; Barton et al. 2004), SDSS quasars (Fan et al. 2003), or gamma-ray burst afterglows (Loeb 2003; Barkana \& Loeb

\footnotetext{
${ }^{1}$ The evidence for a possible Gunn-Peterson (1965) trough in these quasar spectra (White et al. 2003) is still being debated (Songaila 2004).
} 
2004a). Over the next decade, it may also be possible to map directly the neutral hydrogen in the IGM through its $21 \mathrm{~cm}$ line (see recent discussions by Zaldarriaga, Furlanetto, \& Hernquist 2003; Morales \& Hewitt 2003; Loeb \& Zaldarriaga 2003; Gnedin \& Shaver 2003; Sokasian, Furlanetto \& Hernquist 2003) with forthcoming instruments such as LOFAR (http://www.lofar.org/) or SKA (http://www.skatelescope.org/).

A.L. and R.B. acknowledge support by NSF grant AST-0204514 and NATO grant PST.CLG.979414. R.B. is grateful for the support of an Alon Fellowship at Tel Aviv University and of Israel Science Foundation grant 28/02/01. This work was also supported in part by NSF grant AST-0071019 and NASA grant NAG 5-13292 (for A.L.).

\section{REFERENCES}

Barkana, R. \& Loeb, A. 2004a, ApJ, 601, 64

Barkana, R. \& Loeb, A. 2004b, ApJ, in press; astro-ph/0310338

Barton, E. J., Davé, R., Smith, J-D. T., Papovitch, C., Hernquist, L., \& Springel, V. 2004, ApJL, 604, L1

Bromm, V., Kudritzki, R.P. \& Loeb, A. 2001, ApJ, 552, 464

Cen, R. 2003, ApJ, 591, 12

Fan, X. et al. 2003, AJ, 125, 1649

Gnedin, N.Y. 2000, ApJ, 535, 530

Gnedin, N. Y., \& Shaver, P. 2003, ApJ, submitted; astro-ph/0312005

Gunn, J. E., \& Peterson, B. A. 1965, ApJ, 142, 1633

Haiman, Z. 2002, ApJ, 576, L1

Hernquist, L. \& Springel, V. 2003, MNRAS, 341, 1253

Hu, E. M., Cowie, L. L., McMahon, R. G., et al. 2002, ApJ, 568, L75; erratum, ApJ, 576, 99

Hu, E. M., Cowie, L. L., Capak, P., McMahon, R. G., Hayashino, T., \& Komiyama, Y. 2004, AJ, 127,563

Hui, L. \& Haiman, Z. 2003, ApJ, 596, 9

Kneib, J.-P., Ellis, R., Santos, M., Richard, J. 2004, ApJ, in press; astro-ph/0402319

Kodaira, K. et al. 2003, PASJ, 55, L17 
Kogut, A. et al. 2003, ApJS, 148, 161

Leitherer, C., et al. 1999, ApJS, 123, 3

Loeb, A. 2003, to appear in Proc. of IAU Colloquium 192 on "Supernovae", April 2003, Valencia, Spain, eds. J. M. Marcaide and K. W. Weiler; astro-ph/0307231

Loeb, A., \& Zaldarriaga, M. 2003, Phys. Rev. Lett., submitted; astro-ph/0312134

Malhotra, S. \& Rhoads, J. E. 2002, ApJ, 565, L71

Miralda-Escudé, J. 1998, ApJ, 501, 15

Miralda-Escudé, J., Haehnelt, M. \& Rees 2000, ApJ, 530, 1

Morales, M. F. \& Hewitt, J. 2003, ApJ, submitted; astro-ph/0312437

Oh, S. P., Haiman, Z., \& Rees, M. J. 2001, ApJ, 553, 73

Osterbrock, D. E. 1974, Astrophysics of Gaseous Nebulae, W. H. Freeman and Company (San Francisco)

Pello, R., Schaerer, D., Richard J., Le Borgne, J.-F.,\& Kneib, J.-P. 2004, A \& A, 416, L35

Santos, M. R. 2003, MNRAS, submitted; astro-ph/0308196

Santos, M. R., Ellis, R. S., Kneib, J.-P., Richard, J., \& Kuijken, J. 2003, ApJ, submitted; astro$\mathrm{ph} / 0310478$

Scalo, J. 1998, in ASP conference series Vol 142, The Stellar Initial Mass Function, eds. G. Gilmore \& D. Howell, p. 201 (San Francisco: ASP)

Seljak, U., McDonald, P., \& Makarov, A. 2003, MNRAS, in press (astro-ph/0302571)

Sheth, R. K., \& Tormen, G. 1999, MNRAS, 308, 119

Sheth, R. K., \& Tormen, G. 2002, MNRAS, 329, 61

Sokasian, A., Abel, T., Hernquist, L. \& Springel, V. 2003a, MNRAS, 344, 607

Sokasian, A., Abel, T., Hernquist, L. \& Springel, V. 2003b, MNRAS, in press; astro-ph/0307451

Sokasian, A., Furlanetto, S. \& Hernquist, L. 2003, MNRAS, in press; astro-ph/0305065

Songaila, A. 2004, AJ, in press; astro-ph/0402347

Spergel, D. N., et al. 2003, ApJS, 148, 175

Springel, V. \& Hernquist, L. 2003, MNRAS, 339, 312 
Stanway, E. R., et al. 2004, ApJ, 604, L13

Theuns, T., Schaye, J., Zaroubi, S., Kim, T., Tzanavaris, P., \& Carswell, B. 2002, ApJ, 567, L103

Tumlinson, J., Giroux, M. L., \& Shull, J. M. 2001, ApJ, 550, L1

White, R. L., Becker, R. H., Fan, X., \& Strauss, M. A. 2003, AJ, 126, 1

Wyithe, J. S. B. \& Loeb, A. 2003, ApJ, 586, 693

Wyithe, J. S. B. \& Loeb, A. 2004, Nature, 427, 815

Zaldarriaga, M., Furlanetto, S., \& Hernquist, L. 2003, ApJ, submitted; astro-ph/0311514 